\title{
Impact of travel time uncertainties on the solution cost of a two-echelon vehicle routing problem with synchronization
}

\author{
Alexandra Anderluh ${ }^{1}$ (D) Rune Larsen ${ }^{2} \cdot$ Vera C. Hemmelmayr $^{3} \cdot$ Pamela C. Nolz $^{4}$
}

Published online: 22 April 2019

(c) The Author(s) 2019

\begin{abstract}
Two-echelon vehicle routing problems which contain synchronization between vehicles can be deeply impacted by time uncertainty, because one vehicle's delay can propagate to other vehicles. In this paper, we evaluate the deterministic solution of such a problem based on simulated travel time scenarios. The information obtained by simulation is incorporated in the optimization procedure iteratively. Computational results show that the degree of synchronization in an instance is directly correlated with the potential improvements by reoptimization. We present findings on the number of travel time scenarios required to obtain a representative picture of the stochastic solutions. In addition, we demonstrate that time dependent travel times can be aggregated on a city-wide level and linearized as a function of free flow times without major loss of reliability.
\end{abstract}

Keywords Two-echelon vehicle routing · Travel time uncertainty · Synchronization · Simulation-optimization · City logistics

This work is partly funded by the Austrian Research Promotion Agency as part of the Joint Programming Initiative Urban Europe Project CONCOORD (FFG Project No. 839739).

Alexandra Anderluh

alexandra.anderluh@wu.ac.at

1 Research Institute for Supply Chain Management, WU Vienna University of Economics and Business, 1020 Vienna, Austria

2 Management Engineering, DTU Technical University of Denmark, 2800 Kgs. Lyngby, Denmark

3 Institute for Transport and Logistics Management, WU Vienna University of Economics and Business, 1020 Vienna, Austria

4 Center for Mobility Systems, AIT Austrian Institute of Technology, 1210 Vienna, Austria 


\section{Introduction}

Emission-free, agile vehicles are increasingly used for freight deliveries within cities and especially within historic city centers. This development is triggered by the ongoing urbanization as well as the negative effects of climate change caused by greenhouse gas emissions. Also negative health effects in cities caused by other transport-related emissions (WHO 2016) contribute to this trend. However, innovative logistic concepts are required to enable the efficient use of these vehicles within a distribution network. Since the loading capacity and the operating distance of these vehicles are lower, certain limitations have to be taken into consideration. Either consolidation centers near the city center are required, where those vehicles can reload goods (Quak et al. 2014), or a reorganized distribution scheme with synchronization between different types of vehicles can be used to allow for reloading.

The latter approach does not require additional storage facilities, but deals with the problem of reloading agile emission-free vehicles by synchronized meetings with possibly conventional vehicles of larger capacity and operating distance. The conventional vehicles are used for transporting goods from a depot to customers located outside the city center as well as to supply the agile emission-free vehicles at so-called satellites. Satellites are easily accessible spaces which do not offer any storage possibilities. Therefore, temporal and spatial synchronization between reloading vehicles is required. One main characteristic of a solution to such a problem is its inherent interdependence, which makes it sensitive to uncertainties.

The aim of this paper is to investigate the impact of stochastic travel times on the cost of a deterministic solution of a two-echelon vehicle routing problem (2eVRP) with spatial and temporal synchronization. Additionally, we explore how the deterministic solution can be improved with respect to stochasticity by exploiting the available stochastic information. Therefore, we build on an existing solution algorithm for the deterministic problem (Anderluh et al. 2017) and add additional buffer time at time-critical nodes based on information we gain by simulating travel time scenarios.

The contribution of this paper is twofold. On the one hand it provides first insights into the quality of a deterministic solution to a complex VRP with synchronization constraints when applied to a real-life stochastic setting. On the other hand ways of improving the deterministic solution by exploiting stochastic information are evaluated.

The remainder of the paper is organized as follows: Sect. 2 reviews the literature related to stochasticity in routing problems, whereas Sect. 3 describes the problem at hand and Sect. 4 focuses on the solution procedure in use. In Sect. 5 computational results are presented and Sect. 6 concludes the paper.

\section{Literature review}

An early work on stochastic vehicle routing can be found in Stewart and Golden (1983). In the 1990s Bertsimas and van Ryzin (1991), Bertsimas (1992) and Bastian and Rinnooy Kan (1992) continued dealing with this class of problems. 
Gendreau et al. (1996) provided a first literature review on the topic. A recent literature review by Braekers et al. (2016) about VRPs shows that only 58 out of 277 articles published between 2009 and 2015 focus on the stochastic aspects. The majority of these papers-34 articles_deal with stochasticity in demand and dynamic requests. Some of these papers deal with specific solution methods like a branch-cut-and-price algorithm (Gauvin et al. 2014) or a self-adaptive robust search (Chen et al. 2012). Others focus on additional constraints like time windows (Lei et al. 2011), pickup and delivery (Minis and Tatarakis 2011), weighted related cost (Luo et al. 2016) or maximum tour duration (Erera et al. 2010). Juan et al. (2011) generate different scenarios for the VRP with stochastic demand to solve the resulting deterministic problems efficiently with existing solvers. Only 14 articles included in the review by Braekers et al. (2016) deal with stochastic or unknown travel times, only 10 papers focus on time-dependent travel times and none considers stochastic as well as time-dependent travel times at once.

Papers addressing stochastic and dynamic vehicle routing problems of the last three decades have been recently surveyed by Psaraftis et al. (2016), who consider 117 papers in total in their review. 10 papers deal with travel times as dynamic element and 8 papers take travel times as stochastic element into account. Only one out of these papers considers travel times as stochastic and dynamic element simultaneously. Based on Pillac et al. (2013), the difference between stochastic and dynamic problems can be defined as such: Dynamic problems refer to information evolution-i.e. when do we get the information. Stochastic problems refer to information quality-i.e. how reliable is the information at hand.

VRPs solved with stochastic travel times typically take into account time window restrictions as shown in Taş et al. (2014) who solve such a problem with an iterated local search. In some cases, in addition to stochastic travel times and time window restrictions, also stochastic service times are considered (Li et al. 2010; Zhang et al. 2013). Ehmke et al. (2015) focus additionally on the service level of the solution. Besides, Zhang et al. (2012) solve a VRP with simultaneous pickup and delivery while taking into account stochastic travel times, whereas Chen et al. (2014) focus on an arc routing problem with stochastic travel and service times.

The two-echelon capacitated VRP has already been investigated in detail as shown by Cuda et al. (2015) in their survey as well as by Cattaruzza et al. (2017). Synchronization aspects in a deterministic 2eVRP are tackled by Grangier et al. (2016). Stochastic information in a 2eVRP is only taken into account by Tavakkoli-Moghaddam and Raziei (2016) who consider fuzzy demand for multiple products in multiple periods in a two-echelon bi-objective distribution network. To our knowledge there is no paper dealing with stochastic travel times in a 2eVRP.

When dealing with stochastic travel times, one important issue is the probability distribution of such travel times. This distribution can either be determined based on historic empirical data (Aron et al. 2014; Rahmani et al. 2015) or by using 
traffic-related factors like the reduction in traffic capacities because of incidents as well as daily variation in travel activity (Boyles et al. 2010). Although different probability distributions have already been tested in vehicle routing (Gomez et al. 2016; Aron et al. 2014), in the majority of stochastic VRP models a lognormal distribution is assumed as a good approximation (Lecluyse et al. 2009).

In contrast to stochastic travel times, time-dependent travel times can also be taken into account. Gendreau et al. (2017) just recently edit a special section on time-dependent routing in which Montero et al. (2017) focus on a time-dependent TSP with time windows while Mancini (2017) deals with a combined construction heuristic for a time-dependent VRP which includes service times and maximum tour duration as additional constraints. Time-dependent travel times are also considered in green VRPs (Soysal and Cimen 2017) as well as green drone routing problems (Coelho et al. 2017). In addition, time-dependent travel times can also be taken into account for point-to-point evacuation routing and scheduling (Osman and Ram 2017).

Furthermore, Huang et al. (2017) deal with path flexibility in a time-dependent VRP while to our knowledge Soysal et al. (2015) are the only ones who consider time-dependent travel times in a 2eVRP.

Wang et al. (2016) evaluate the value of deterministic solutions in service network design. They show that parts of deterministic service network design solutions can be used effectively in a stochastic setting. To our knowledge the basic idea of their work has not been applied to a 2 eVRP with spatial and temporal synchronization yet.

Therefore, in this paper we focus on the impact of stochastic travel times on the solution cost of a 2eVRP with synchronization and customer deliveries on both echelons. Our aim is to gain insights into the impact of these uncertainties to the deterministic solution of the problem based on fixed as well as time-dependent travel times and to evaluate methods of reoptimizing the deterministic solution based on scenario simulation of lognormally distributed travel times.

\section{Problem description}

In this paper we consider a 2 eVRP with spatial and temporal synchronization, and customer deliveries on both echelons. Customers are divided into two groups. Customers located in the predefined city center have to be served by cargo bikes, whereas all other customers have to be served by vans. Vans as first echelon vehicles start their routes at the van depot at the outskirts of the city (see red large rectangle in Fig. 1), which is also the storage facility for the goods.

On the second echelon, cargo bikes start their routes at the bike depot inside the city center (see green small rectangle in Fig. 1). This bike depot is only used for parking and has no storage facilities. Thus, a cargo bike requires at least one temporally and spatially synchronized meeting with a van at one of the predefined synchronization points, the set of satellites (see blue triangles in Fig. 1), right after 


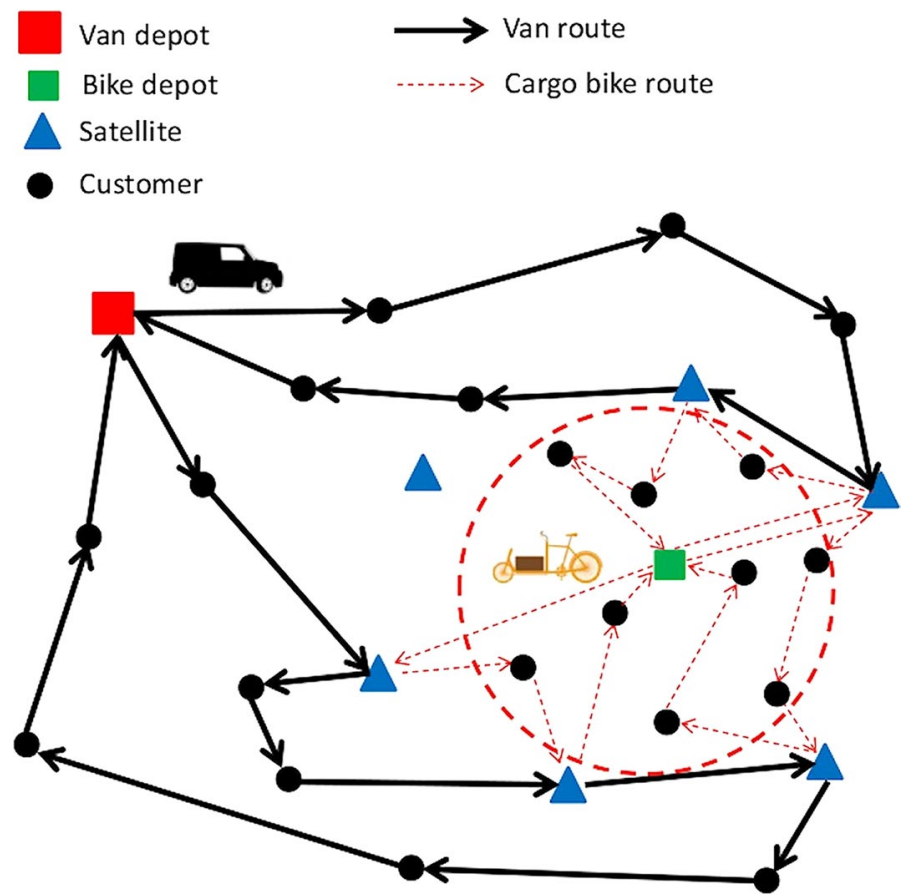

Fig. 1 City distribution scheme with vans and cargo bikes

starting from the bike depot. Because of a cargo bike's limited capacity, reloading may also be required, once the cargo bike runs out of cargo during its tour (see cargo bike trips (dotted lines in Fig. 1) starting from the bike depot (green small rectangle), meeting a van at a satellite (blue triangle) to get the first load, then serving customers (black dots) and visiting again a satellite for loading the next part of goods).

The overall objective of this optimization problem is to minimize total cost for transporting all ordered goods to the customers either by van or by bike. This total cost consists of distance-based vehicle cost, time-based personnel cost and fixed cost for each vehicle in use. In addition to a maximum allowed route duration, synchronized meetings between vans and cargo bikes at satellites are modeled in a way that any vehicle involved in a synchronization must not wait longer at a satellite than a predefined fraction of the maximum allowed route duration. Capacity constraints for vans on the first echelon must not be exceeded during each van route, whereas capacity constraints for bikes on the second echelon become effective on any route segment between two satellite visits, as well as the last part of the bike route from a satellite to the bike depot. For the complete mathematical model the interested reader is referred to Anderluh et al. (2017). 

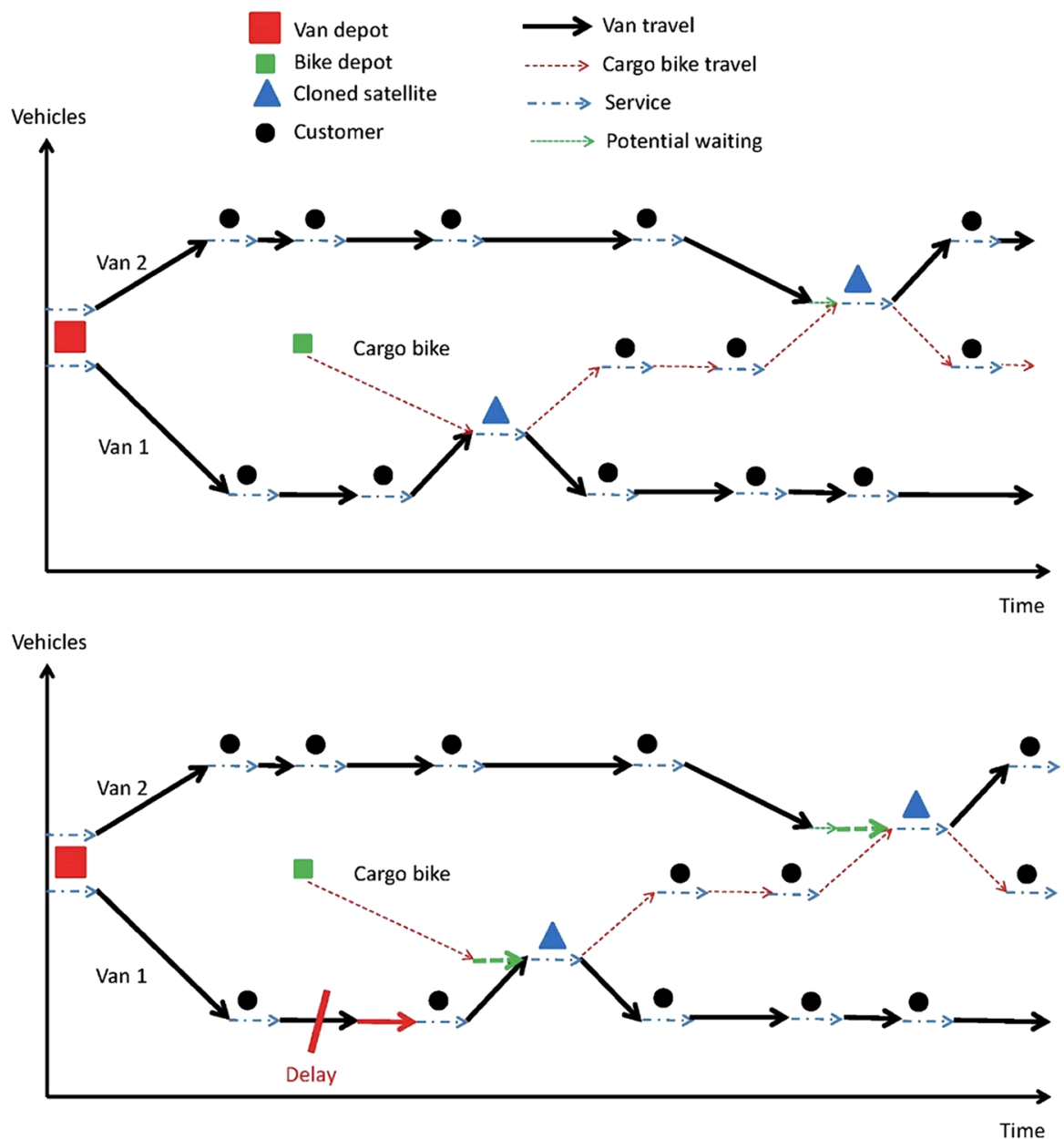

Fig. 2 Interdependence between routes because of synchronization (upper part) and potential impact of a delay (lower part)

The multiple synchronizations between vans and cargo bikes in the described city distribution scheme, create a high degree of interdependence between the routes. A cargo bike can meet with a van to get the first part of goods, deliver it and then meet with another van to get the next load (see upper part in Fig. 2).

A delay of one vehicle naturally has an impact on the vehicle itself (see Van 1 in the lower part of Fig. 2) but-because of its synchronized meeting with another vehicle-it can also cause delays at that second vehicle (see Cargo bike in the lower part of Fig. 2). Furthermore, the delay can also propagate to other vehicles which have a synchronized meeting with any of the already affected vehicles (see Van 2 in the lower part of Fig. 2). In the end all vehicles displayed in Fig. 2 are affected by only one delay. 
Therefore, it is of interest how such a deterministic solution behaves under more realistic conditions characterized by uncertainties. In this context, we explicitly investigate the impact of stochastic travel times on the deterministic solution, because travel time deviations are constantly caused by different amounts of traffic, congestion, and/or traffic jams.

In this paper we rely on findings by Lecluyse et al. (2009) for the probability distributions of travel times and assume a lognormal distribution for the travel times of vans. For extensive information on determining appropriate travel time distributions we refer the interested reader to Gomez et al. (2016) and Aron et al. (2014).

Travel times of bikes are assumed as rather unaffected by congestion and hardly uncertain as shown for example by Jensen et al. (2010).

\section{Solution procedure}

For the deterministic problem described in Sect. 3 a two-stage GRASP with path relinking is used (see Pseudocode 1, line 1), where cargo bike routes and van routes are built sequentially. After building the cargo bike routes, improving them by local search with a 2opt-, a move-, and a swap-operator and inserting necessary satellites for synchronization purposes, the van routes are built accordingly, based on the demand and synchronization information of the already fixed cargo bike routes. Path relinking then enables finding additional promising solutions by converting one solution out of a pool of solutions into the pre-selected so-called guiding solution (Anderluh et al. 2017).

Then we apply a Monte Carlo simulation to generate $N$ travel time scenarios for the deterministic solution based on lognormally distributed travel times. This yields different costs $C_{\text {stoch }}^{s}$ for each scenario $s$. Based on these simulations we then calculate the average cost of stochastic travel times $\bar{C}_{\text {stoch }}=\frac{1}{N} \sum_{s=1}^{N} C_{s t o c h}^{s}$ of the solution (see Pseudocode 1, lines 2-3) to estimate the performance of the deterministic solution under uncertain travel times. Hence, the higher the average cost of stochastic travel times $\bar{C}_{\text {stoch }}$ in proportion to the cost of the deterministic solution $C_{d e t}$, the worse the performance of the deterministic solution under travel time uncertainties. Therefore, the focus of this work is to find a good way of reducing $\bar{C}_{\text {stoch }}$ without increasing $C_{d e t}$ significantly. 


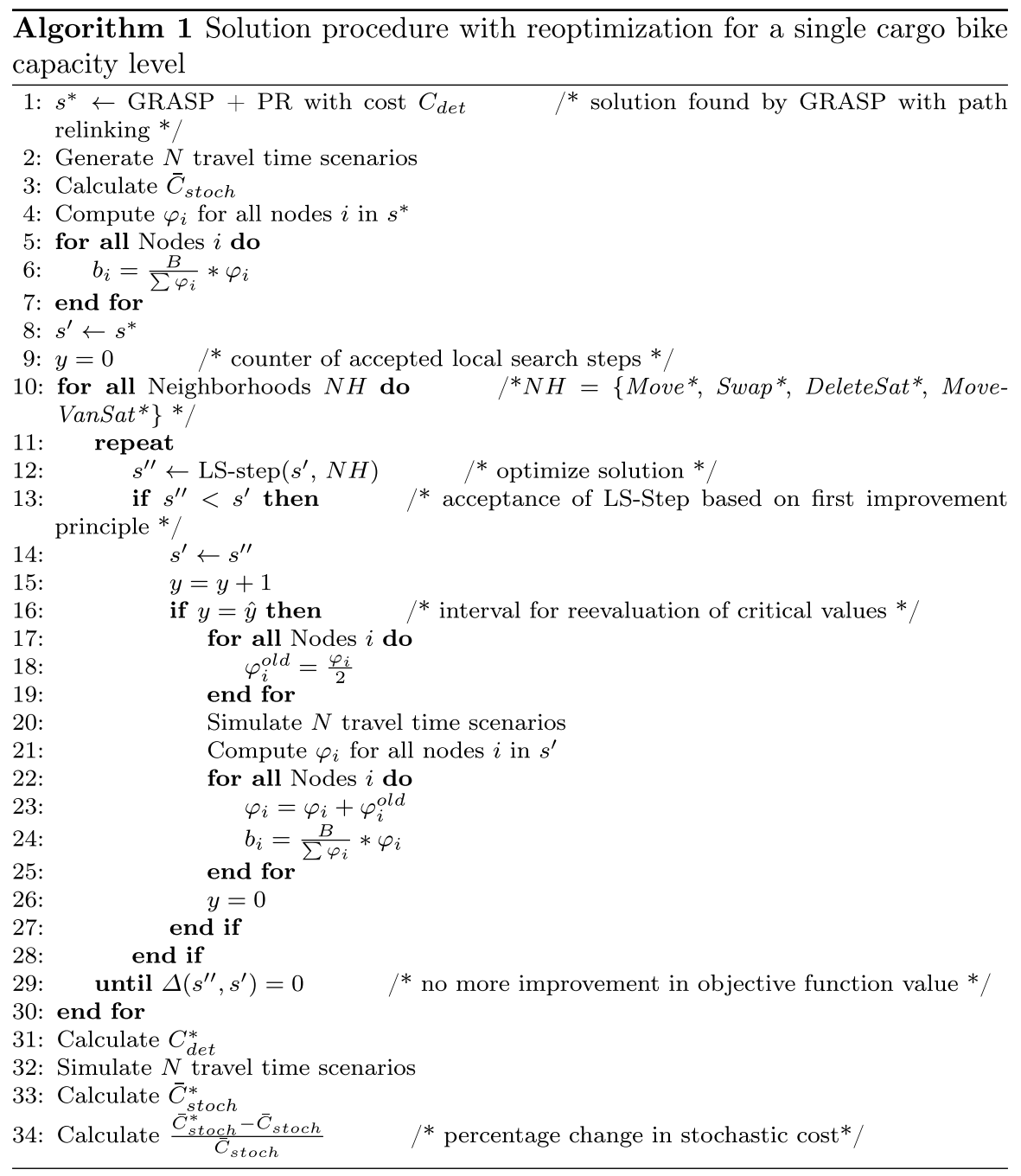

After the average cost of stochastic travel times $\bar{C}_{\text {stoch }}$ is determined, we mark in all simulated travel time scenarios infeasible paths with respect to the maximum route duration $t_{\max }$, which is assumed as hard constraint. Such a path is called critical path.

For each node $i$ we evaluate the critical value $\varphi_{i} \mid 0 \leq \varphi_{i} \leq 1$, which represents the fraction of scenarios in which node $i$ is part of a critical path (see Pseudocode 1, line 4). Then we assign an additional buffer time $b_{i}$ to the given service time $\lambda_{i}$ of a critical node, i.e. a node $i$ where $\varphi_{i}>0$ (see Pseudocode 1, lines 5-7). Then this new deterministic problem is reoptimized (see Pseudocode 1, lines 10-30).

The reoptimization phase of the deterministic solution now requires additional local search operators which respect all synchronization constraints of the existing deterministic solution - that is the required spatial and temporal synchronization of vehicles at 
satellites and the related maximum allowed waiting time for vehicles at satellites traced through the complete solution:

- A Move*-operator, which moves one customer to a position in one of the routes of the same type with respect to the synchronization constraints.

- A $S w a{ }^{*}$-operator, which exchanges two customers in routes of the same type with respect to the synchronization constraints.

- A MoveVanSat*-operator, which inserts available satellites in van routes with respect to the synchronization constraints. This includes also changing the satellites in the respective bike routes.

- A DeleteSat*-operator, which removes unnecessary satellites from the solution.

Conducted pretests show that the most preferable order of applying these operators is:

1. Move*-operator

2. Swap*-operator

3. DeleteSat*-operator

4. MoveVanSat*-operator

In this way, the $S w a{ }^{*}$-operator which searches a larger neighborhood than the Move*-operator, is applied second. The DeleteSat*-operator is used to remove unnecessary satellites from the solution after applying the former two operators. Then the MoveVanSat*-operator is applied to check if satellites can be shifted in the solution.

The re-evaluation of the critical paths is done regularly after each $\hat{y}$ th accepted local search step (see Pseudocode 1, lines 13-28). This reassessment of critical paths is based on simulated travel time scenarios and yields new $\varphi_{i}$ values (see Pseudocode 1 , line 21 ). Old $\varphi_{i}$-values are halved and stored (see Pseudocode 1, lines 17-19). Then the new values for $\varphi_{i}$ are summed up with the old ones and new values for $b_{i}$ are calculated (see Pseudocode 1, lines 22-25). This is done to incorporate a kind of memory of the critical nodes during the reoptimization process. Finally, we re-evaluate the average cost of stochastic travel times of the new found solution $\bar{C}_{\text {stoch }}^{*}$ without buffer times and compare it with the respective average cost $\bar{C}_{\text {stoch }}$ calculated before (see Pseudocode 1, lines 31-34).

Table 1 provides an overview of all parameters used in this paper.

\section{Computational results}

The algorithm was coded in $\mathrm{C} / \mathrm{C}++$. All tests were performed under Linux Ubuntu 16.04 LTS on a Virtual Machine using 2 processors and 2GB memory on a host Intel(R) Core(TM) i5-3320 M CPU @ 2.60GHz 4GB RAM and running on a single thread.

Because of the different scale of the objective function values of our test instances, we focus in our tests on the relative change in average cost of stochastic travel times of the reoptimized solution $\bar{C}_{\text {stoch }}^{*}$ compared to the respective cost 
Table 1 Overview of parameters and variables

\begin{tabular}{ll}
\hline $\begin{array}{l}\text { Parameter/ } \\
\text { variable }\end{array}$ & Description \\
\hline$B$ & Total amount of buffer time \\
$b_{i}$ & Fraction of buffer time $B$ assigned to node $i$ \\
$t_{\text {max }}$ & Maximum allowed route duration \\
$\varphi_{i}$ & Critical value of node $i$ \\
$d_{i}$ & Demand of node $i$ \\
$\lambda_{i}$ & Service time at node $i$ \\
$V_{c}^{b}$ & Set of customers to be served by a second echelon vehicle \\
$Q_{\text {min }}^{b}$ & Minimum capacity of a second echelon vehicle \\
$Q_{\text {max }}^{b}$ & Maximum capacity of a second echelon vehicle \\
$Q_{z}^{b}$ & Capacity of a second echelon vehicle at capacity level $z$ \\
$Q^{v}$ & Capacity of a first echelon vehicle \\
$C_{d e t}$ & Cost of original solution based on deterministic travel times \\
$C_{s t o c h}^{s}$ & Cost of original solution based on travel times generated in scenario $s$ \\
$\bar{C}_{s t o c h}$ & Average cost of the original solution evaluated averaged over the $N$ generated scenarios \\
$C_{d e t}^{*}$ & Cost of reoptimized solution based on deterministic travel times \\
$\bar{C}_{s t o c h}^{*}$ & Average cost of the reoptimized solution evaluated averaged over the $N$ generated scenarios \\
$\hat{y}$ & Number of accepted LS-steps after which the recalculation of $\varphi_{i}$ is applied \\
\hline &
\end{tabular}

of the original deterministic solution $\bar{C}_{\text {stoch }}$ (see Sect. 4 for details). Therefore, we look at $\frac{\overline{\bar{C}}_{\text {stoch }}^{*}-\bar{C}_{\text {stoch }}}{\bar{C}_{\text {stoch }}}$ to assess the performance of the reoptimized solutions.

The instances used and data sets generated during the current study are available from the corresponding author on request.

The remainder of the section starts with a detailed description of the test instances used, followed by results of pretests and parameter tuning. Then the computational results regarding the degree of synchronization of an instance are discussed. Eventually, the use of time-dependent travel times is investigated.

\subsection{Test instances}

For our computational tests we use three types of instances. Six adapted Solomon instances (one out of each group of Solomon instances) are used for pretests and parameter tuning. The adaption of the Solomon instances by Grangier et al. (2016) is extended by using the Solomon depot as bike depot and by adding additional vehicle information to reflect our two assumed vehicle types.

In addition, 12 synthetic test instances which cover three different layouts and two different instance sizes are generated. The three different layouts are based on the idea of the Solomon instances with randomly located customers $(r)$, clustered customers $(c)$, and a combination of the former two customer location schemes $(r c)$. All customers are located in an area representing a city like Vienna. The instances 
include 100/125 ( $n 100, n 125)$ customers and 10 satellites. The satellites are located around the assumed city center approximately in the middle of the instance area. The values for the vehicles used reflect realistic values for speed, capacity as well as costs of cargo bikes and 3.5-ton vans. Service time and demand of customers are random numbers in an appropriate range (for further details see Anderluh et al. (2017)).

To cover also time-dependent travel times a real-life instance of the city of Vienna is used. This instance includes 100 randomly selected pharmacies in Vienna and the van depot is the location of a pharmacy wholesaler in Vienna. 18 potential satellites are assumed at appropriate locations along the outer ring street. The bike depot is assumed at an appropriate location in the city center (for further details see Anderluh et al. (2017)). We expand this instance by time-dependent travel times for the first echelon vehicles (vans) based on data described as follows.

Leodolter et al. (2015) use historical floating car data (FCD) collected from 3500 taxis in the region of Vienna. The taxis are equipped with an on-board-unit providing global positioning system (GPS) data of the position of the taxis every 30 to $60 \mathrm{~s}$. The GPS data is analyzed using the real-world FCD system FLEET (Toplak et al. 2011) and projected on the road network graph in order to obtain vehicle speed measurements.

The goal of the work of Leodolter et al. (2015) is to estimate the variation of travel speed in the course of a typical day. The authors present a method using a linear regression model based on static map parameters to estimate the diurnal variation of travel times for vehicles in urban areas. This enables the estimation of realistic travel times from static map coefficients without the need for further data collection. Data is extracted from the FLEET system, containing daily time series of 96 15-min intervals for 4 different speed profiles on each link of the road network of Vienna (Toplak et al. 2011).

\subsection{Pretests and parameter settings}

To figure out an appropriate number of scenarios for each evaluation step, we conduct pretests with an increasing number of scenarios. In these tests a sample size of $N=60$ yields already an acceptably low deviation in the simulated travel time scenarios, which we express in the additional average cost of stochastic travel times $\left(\bar{C}_{\text {stoch }}-C_{d e t}\right)$ in cost units. Furthermore, this sample size also guarantees good results in a reasonable amount of computational time (see Fig. 3).

Three different methods for allocating buffer times are tested. The total amount of buffer time $B$, which is a fraction of the maximum route duration $t_{\max }$, is assigned to each node $i$ :

(1) Proportionally based on $\varphi_{i}$.

(2) As equal share to any node $i$ with $\varphi_{i}>0.5$.

(3) As a combination of the former two ways. $2 / 3$ of $B$ are based on (1), the rest is based on (2).

The reassignment of $B$ is done either only once before starting the reoptimization or after every $\hat{y}$ th accepted improvement step in the local search of the reoptimization with $\hat{y} \in(5000,1000,500,100,10)$. 


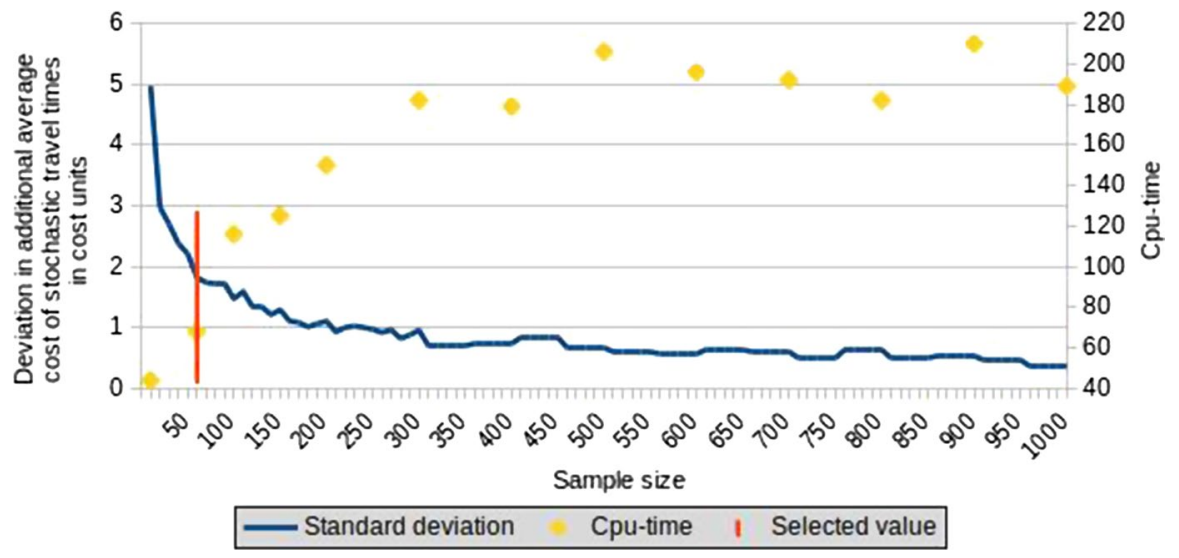

Fig. 3 Impact of sample size of scenario simulation on deviation in additional average cost of stochastic travel times (in cost units) and average cpu-time (in s)

Table 2 Ranking of buffer time assignment type and reoptimization mode with respect to change in average cost of stochastic travel times

\begin{tabular}{lllllll}
\hline $\begin{array}{l}\text { Assign- } \\
\text { ment } \\
\text { type }\end{array}$ & \multicolumn{6}{l}{ Reoptimization mode } \\
\cline { 2 - 7 } & Once & $\hat{y}=5000$ & $\hat{y}=1000$ & $\hat{y}=500$ & $\hat{y}=100$ & $\hat{y}=10$ \\
\hline$(1)$ & 0.765 & 0.984 & $\mathbf{1 . 0 0 0}$ & 0.921 & 0.890 & 0.881 \\
$(2)$ & 0.881 & 0.899 & 0.914 & 0.817 & 0.754 & 0.831 \\
$(3)$ & 0.767 & 0.818 & 0.944 & 0.884 & 0.905 & 0.867 \\
\hline
\end{tabular}

Bold values indicate the best setting

The different parameter settings were tested with all pretest instances and an average of five runs was considered for each instance. Table 2 shows the ranking of all combinations with respect to change in average cost of stochastic travel times, where 1.000 as the maximum value represents the best parameter setting.

The tests show that using type (1) for the assignment of buffer time $B$ and reoptimizing the critical values $\varphi_{i}$ after every 1000th acceptance of a local search step yield the best results.

Nevertheless, the impact of the assignment type of $B$ is rather small. Furthermore, the assessment of different reoptimization modes depicted in Table 2 show that calculating the critical values $\varphi_{i}$ after every 1000th acceptance step in the local search yields the best results for each assignment type of $B$. Doing the reoptimization only once at the beginning performs worst for assignment types (1) and (3), while assignment type (2) gives the worst result while reoptimizing after every 100th acceptance step. In all cases reoptimizing after every 5000th, 500th, 100th or 10th acceptance step in the local search does not improve the results, although no clear pattern can be distinguished. In addition, computational times for all settings show that $\hat{y}=1000$ yields good results in a small amount of computational time (see Fig. 4).

To determine an appropriate value for $B$, which we assume as fixed value, tests for different percentages of $t_{\max }(5,10,15,20$ and 25) over all test instances have 


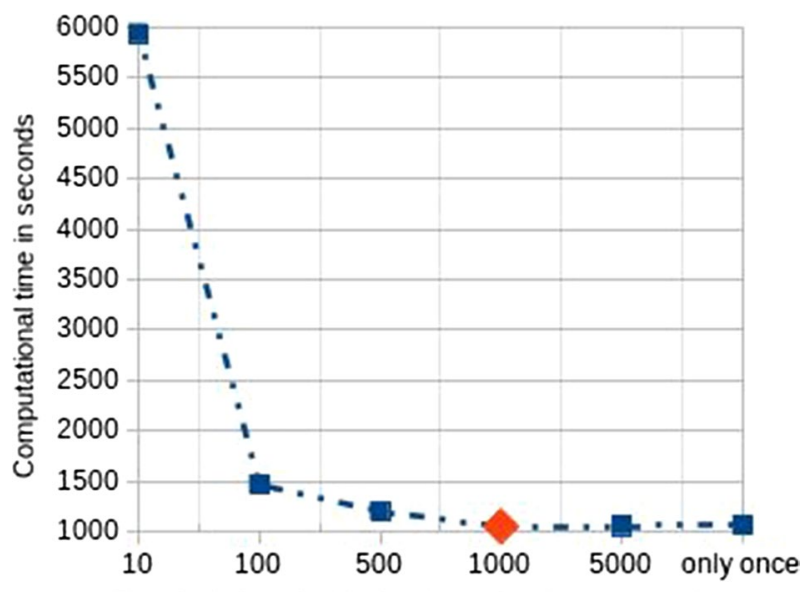

Recalculation of critical values after ŷ local search steps

Fig. 4 Average computational time for recalculation of critical values after $\hat{y} \in(10,100,500,5000)$ local search steps and only once at the beginning

been conducted. Figure 5 depicts the increase from the basic deterministic $\operatorname{cost} C_{d e t}$ to the resulting deterministic cost $C_{d e t}^{*}$ after reoptimization versus the decrease from the average cost of stochastic travel time $\bar{C}_{\text {stoch }}$ compared to the cost $\bar{C}_{\text {stoch }}^{*}$ resulting after reoptimizing the solution. Hence, $B=\frac{t_{\max }}{10}$ gives a good compromise between those two indicators.

\subsection{Impact of the degree of synchronization}

The level of interdependence in a solution depends on several drivers like the number of customers in the city center, the demand of these customers, and the specific location of these customers. These factors impact the total load of cargo bikes, and such the required number of synchronized meetings with vans. One of the main influencing factors is the degree of synchronization in a problem instance. This factor is strongly correlated with $Q_{z}^{b}$ - the capacity of the second echelon vehicles (cargo bikes) which reflects a certain synchronization level $z$. We performed our computational tests with a capacity varying from a minimum required capacity $Q_{\min }^{b} \geq \max \left(d_{i}\right) \forall i \in V_{c}^{b}$ to a maximum capacity $Q_{\max }^{b} \leq \frac{Q^{v}}{2}$. $Q_{\min }^{b}$ represents the highest demand of all customers supplied by a second echelon vehicle in the instance, as every customer has to be served by exactly one visit. $Q_{\max }^{b}$ is assumed as half of the first echelon vehicle's capacity. Between these two bounds the capacity $Q_{z}^{b} \mid z=\{1(=\min ), 2, \ldots, n(=\max )\}$ is then increased by $Q_{z}^{b}=Q_{z-1}^{b}+\frac{Q^{v}}{20} \forall\{z \mid 1<z<n\}$.

A major insight from our computational results is that the amount of the decrease in average cost of stochastic travel times clearly depends on the degree of synchronization which is reflected by $Q_{z}^{b}$. Lower capacity and hence the necessity of more synchronizations (see dashed line in Fig. 6) for a feasible solution of the problem is related to a stronger decrease in average cost of stochastic travel times as depicted by the solid line in Fig. 6 . 
Fig. 5 Trade-off between increase in deterministic cost and decrease in average cost of stochastic travel times depending on the amount of $B$ as fraction of $t_{\max }$

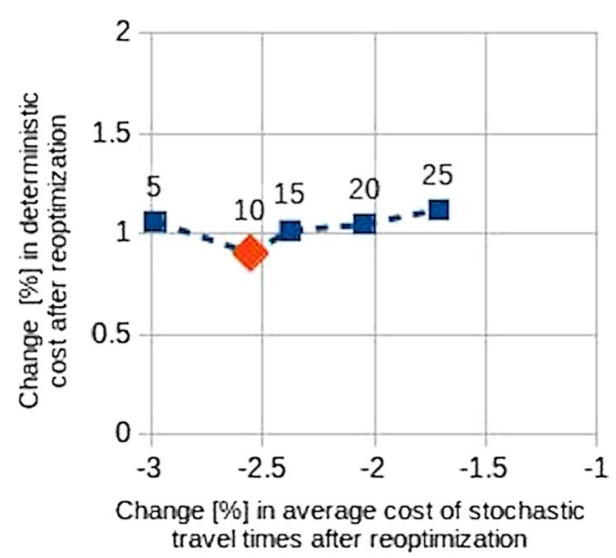

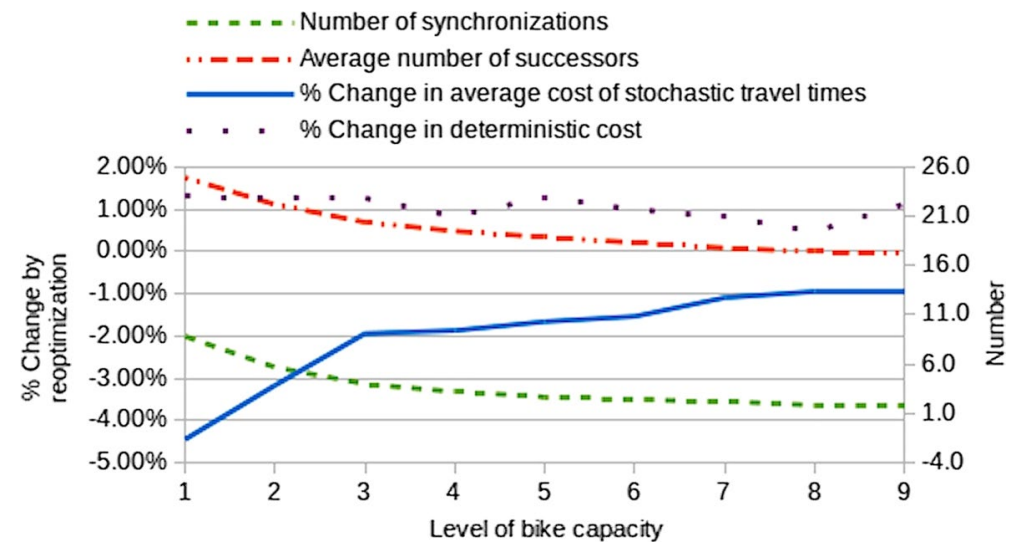

Fig. 6 Impact of level of bike capacity on change in average cost of stochastic travel times (\%), number of required synchronizations and average number of successors

A low level of bike capacity also implies a higher average number of successors (see dash-dotted line in Fig. 6), which reflects the average number of nodes in an instance that follows directly (in the same route) or indirectly (in a synchronized route) each node of the instance. These findings hold for all our test instances.

Figure 6 also shows that for low bike capacity levels 1 and 2-which reflect a high degree of synchronization-the average cost of stochastic travel times can be reduced by the reoptimization step by around $4 \%$ on average over all test instances. From bike capacity levels 3-6 this reduction is around $2 \%$ and for bike capacity levels 7-9 this reduction stabilizes around $1 \%$.

Therefore, our results show that the more interdependent the instance is, the more the performance of the deterministic solution under travel time uncertainties can be improved by reoptimization.

On the other hand the increase in deterministic cost by reoptimization is rather constant at around $1 \%$ on average for all bike capacity levels (see dotted line in Fig. 6).

Detailed results for each instance and bike levels are depicted in Tables 3, 4 and 5 in the "Appendix". These results show that, especially for the clustered test instances 
(n*_c*, and 'vienna'), the average cost of stochastic travel times can be reduced by up to around $9 \%$, whereas instances with randomly distributed customers $\left(n^{*} \_r^{*}\right)$ in most cases perform worse in our setting.

In contrast to that the size of the instance does not show a significant impact in our tests.

\subsection{Use of time-dependent Travel Times}

For the Vienna test instance we test also the influence of time-dependent travel times in the reoptimization process. Therefore, we calculate solutions for starting times of first echelon vehicles from 7:00 to 13:00 in 1-h steps. We compare these results with the solution calculated based on free flow times.

Additionally, we use the idea of linear regression (Leodolter et al. 2015) based on time-dependent travel times in the provided 1-h slots from 7:00 am to 7:00 pm on typical working days. Comparing free flow times for all arcs in the Vienna instance with the average time based on the time-dependent times over the working day is depicted in Fig. 7. Therefore, recalculating travel times based on free flow times with the above mentioned linear regression seems to be a viable estimate.

Travel times for the second echelon vehicles (cargo bikes) are assumed as steady over the working day as bikes are usually not delayed by accidents or traffic jams. This assumption is also supported by Jensen et al. (2010), who show in their survey that cyclists travel at nearly the same speed during the day.

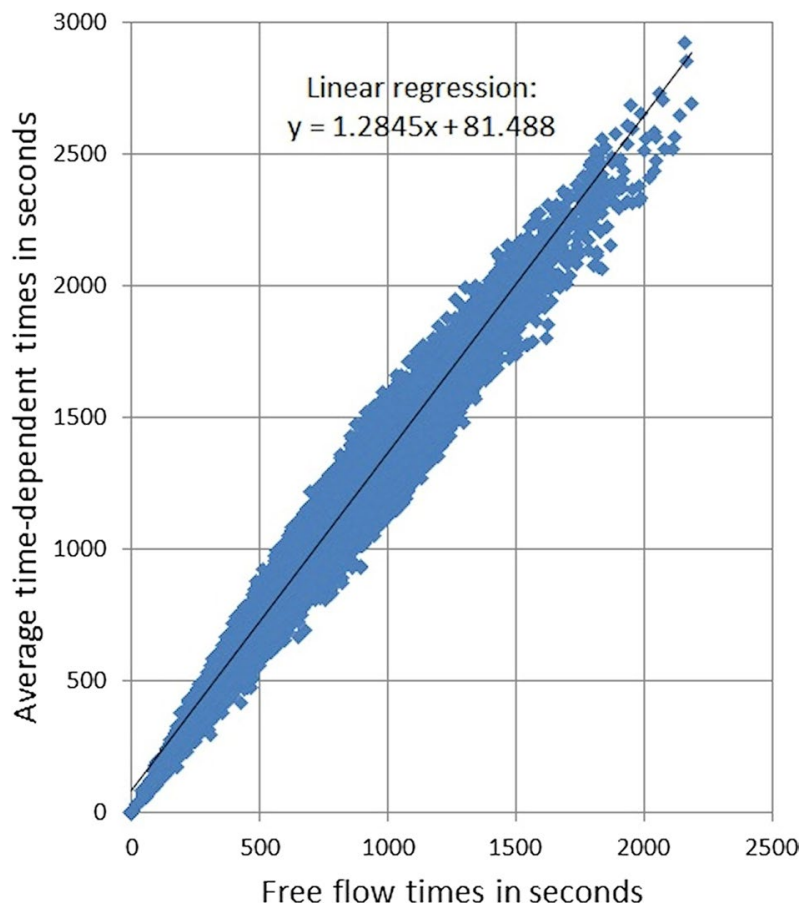

Fig. 7 Linear regression of average time-dependent travel times to free flow times 


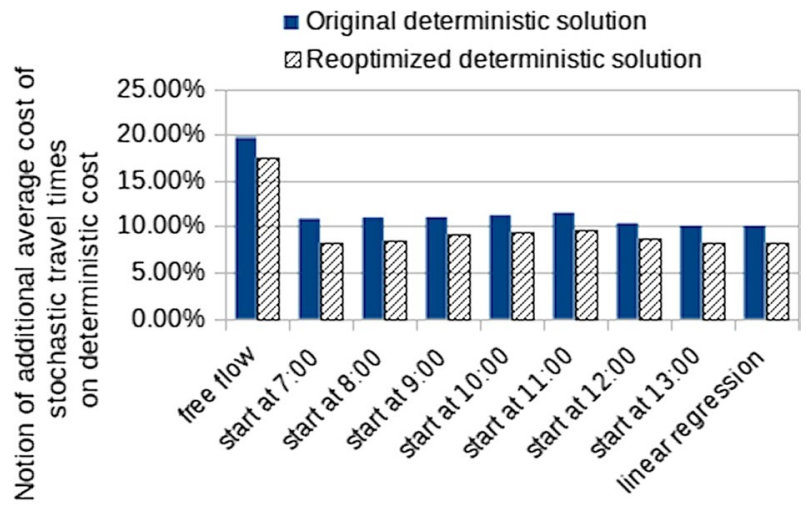

Travel time basis

Fig. 8 Notion of additional average cost of stochastic travel times on cost of deterministic original and reoptimized solution calculated for different travel time basis

Figure 8 depicts the notion of additional average cost of stochastic travel times $\left(\bar{C}_{\text {stoch }}-C_{d e t}\right)$ on the deterministic $\operatorname{cost} C_{d e t}$ for the original deterministic solution (solid bar) as well as the respective cost $\left(\bar{C}_{\text {stoch }}^{*}-C_{d e t}^{*}\right)$ as fraction of $C_{d e t}^{*}$ (hatched bar). The consistent difference between the solid and the hatched bars for each travel time basis show that reoptimization works comparably well regardless of the travel time basis.

Furthermore, Fig. 8 shows the comparable behavior when using time-dependent travel times as well as travel times gained by applying linear regression. On the other hand the use of free flow travel times causes proportionally higher additional cost of stochastic travel times.

Based on these results we conclude that, at least for our realistic test instance of the city of Vienna, using adapted free flow times performs similarly to using timedependent travel times. This is of importance because deriving time-dependent travel times based on floating car data for the complete road network requires significantly more effort than determining free flow travel times from publicly available sources as for example openstreetmap.org, surveying time-dependent data only for some representative arcs and deriving the linear regression based on these observations.

\section{Conclusion}

In this paper we analyze the performance of an innovative city distribution scheme for a two-echelon vehicle routing problem with temporal and spatial synchronization between vans and cargo bikes in a realistic setting. Travel time uncertainties are taken into account.

We present a reoptimization method for the deterministic solution of the problem based on stochastic information to improve the quality of the overall solution. Computational results show that our simple iterative reoptimization of the deterministic solution based on information gained by evaluating simulated travel time scenarios can improve the behavior of the solution under real-life circumstances significantly. 
The evaluation of all test instances shows that the performance of our method depends on the degree of synchronization - that is the number of synchronizations between firstechelon and second-echelon vehicles - in the solution. The higher the degree of synchronization the more can be gained by applying the reoptimization step. We expect this to generalize to other vehicle routing problems containing synchronization constraints.

Using time-dependent travel times has turned out to yield no better results than transformed free flow travel times, where the transformation is based on a simple linear regression. These results indicate the good performance of the latter approach, which is of importance especially for decision makers, because such travel times are easier to obtain than time-dependent ones.

Additional stochastic information concerning for example uncertain service times as well as dynamic requests are one direction for future research.

To cover the main advantages of the proposed cargo bikes-especially their lack of local emissions and of noise-future research should also take into account the evaluation of emissions, like greenhouse gases, particulate matters and nitrogen oxides, as well as the evaluation of other external factors, like noise, side-effects of congestion and accidents within the optimization process.

Acknowledgements Open access funding provided by Vienna University of Economics and Business (WU). We thank Markus Straub from AIT for his help with calculating time-dependent travel times for our Vienna instance, and we gratefully acknowledge the data provided by Taxi 31300 .

Open Access This article is distributed under the terms of the Creative Commons Attribution 4.0 International License (http://creativecommons.org/licenses/by/4.0/), which permits unrestricted use, distribution, and reproduction in any medium, provided you give appropriate credit to the original author(s) and the source, provide a link to the Creative Commons license, and indicate if changes were made.

\section{Appendix}

\section{Description of columns}

Inst: Instance

$Q_{z}$ : Bike capacity level

Synchs: Number of synchronizations

Succs: Average number of successors

$$
\begin{aligned}
\Delta C_{\text {stoch }} & =\frac{\bar{C}_{\text {stoch }}^{*}-\bar{C}_{\text {stoch }}}{\bar{C}_{\text {stoch }}} \\
\Delta C_{d e t} & =\frac{C_{d e t}^{*}-C_{d e t}}{C_{d e t}}
\end{aligned}
$$

$\Delta$ Time: Difference in total time required between deterministic and reoptimized solution

$\Delta$ Dist: Difference in total distance required between deterministic and reoptimized solution 
Table 3 Detailed results for instance 'Vienna'

\begin{tabular}{llllllll}
\hline Inst & $Q_{z}$ & Synchs & Succs & $\Delta C_{\text {stoch }}[\%]$ & $\Delta C_{\text {det }}[\%]$ & $\Delta$ Time $[\%]$ & $\Delta$ Dist [\%] \\
\hline Vienna & 1 & 10.2 & 32.9 & -7.52 & 0.99 & 2.16 & 5.52 \\
& 2 & 6.0 & 28.3 & -3.46 & 1.15 & 1.74 & 6.82 \\
& 3 & 5.0 & 26.6 & -3.04 & 1.32 & 3.23 & 6.67 \\
& 4 & 4.0 & 25.7 & -5.07 & 1.26 & 3.82 & 6.70 \\
& 5 & 3.0 & 24.4 & -3.50 & 0.58 & 1.03 & 5.63 \\
& 6 & 3.0 & 23.4 & -3.33 & 0.85 & 4.82 & 3.74 \\
& 7 & 2.0 & 20.2 & -0.67 & 0.25 & 0.20 & 1.55 \\
& 8 & 2.0 & 20.9 & -0.58 & 0.68 & 1.08 & 4.96 \\
& 9 & 2.0 & 20.5 & -0.96 & 1.13 & 1.90 & 6.95 \\
\hline
\end{tabular}

Table 4 Detailed results for instances ' $100 *$,

\begin{tabular}{|c|c|c|c|c|c|c|c|}
\hline Inst & $Q_{z}$ & Synchs & Succs & $\Delta C_{\text {stoch }}[\%]$ & $\Delta C_{d e t}[\%]$ & $\Delta$ Time $[\%]$ & $\Delta$ Dist [\%] \\
\hline \multirow[t]{9}{*}{ n100_c1 } & 1 & 9.6 & 22.9 & -6.08 & 1.32 & 0.53 & 8.52 \\
\hline & 2 & 6.4 & 19.4 & -4.44 & 0.38 & 0.11 & 3.15 \\
\hline & 3 & 4.0 & 17.7 & -2.07 & 0.83 & 2.39 & 3.87 \\
\hline & 4 & 3.0 & 16.2 & -3.23 & 1.11 & 2.74 & 4.94 \\
\hline & 5 & 3.0 & 16.7 & -1.09 & 0.75 & 0.14 & 4.95 \\
\hline & 6 & 3.0 & 16.9 & -2.78 & 1.06 & 0.97 & 5.89 \\
\hline & 7 & 2.2 & 15.9 & -0.75 & 0.72 & 1.91 & 3.06 \\
\hline & 8 & 2.0 & 15.6 & -0.28 & 1.04 & 3.15 & 3.77 \\
\hline & 9 & 2.0 & 15.7 & -0.81 & 0.33 & 0.34 & 1.59 \\
\hline \multirow[t]{9}{*}{ n100_c2 } & 1 & 12.0 & 25.5 & -5.12 & 0.42 & 0.10 & 2.81 \\
\hline & 2 & 7.4 & 20.9 & -5.35 & 0.45 & 0.35 & 3.33 \\
\hline & 3 & 5.0 & 18.9 & -1.14 & 0.18 & 0.40 & 1.22 \\
\hline & 4 & 4.0 & 17.8 & -1.48 & 0.98 & 3.85 & 3.91 \\
\hline & 5 & 3.2 & 16.3 & -1.45 & 0.49 & 1.47 & 1.70 \\
\hline & 6 & 3.0 & 16.3 & -2.12 & 1.00 & 2.71 & 3.80 \\
\hline & 7 & 3.0 & 16.4 & -2.01 & 1.09 & 1.40 & 6.01 \\
\hline & 8 & 2.0 & 14.8 & -0.83 & 0.80 & 0.82 & 4.71 \\
\hline & 9 & 2.0 & 14.5 & -2.19 & 0.72 & 2.07 & 3.39 \\
\hline \multirow[t]{9}{*}{ n100_r1 } & 1 & 7.0 & 18.8 & -5.58 & 1.14 & 1.83 & 6.16 \\
\hline & 2 & 5.2 & 17.6 & -2.35 & 1.20 & 0.38 & 7.60 \\
\hline & 3 & 3.8 & 17.2 & -0.75 & 1.14 & 1.24 & 6.67 \\
\hline & 4 & 3.0 & 16.4 & -0.98 & 0.75 & 2.27 & 3.75 \\
\hline & 5 & 2.0 & 16.2 & -2.25 & 1.64 & 5.89 & 6.57 \\
\hline & 6 & 2.0 & 15.4 & -4.01 & 1.02 & 2.80 & 5.07 \\
\hline & 7 & 2.0 & 15.2 & -0.22 & 1.37 & 4.54 & 5.73 \\
\hline & 8 & 2.0 & 15.4 & -2.53 & 1.58 & 5.60 & 6.32 \\
\hline & 9 & 2.0 & 15.6 & -0.58 & 0.76 & 2.38 & 3.49 \\
\hline
\end{tabular}


Table 4 (continued)

\begin{tabular}{|c|c|c|c|c|c|c|c|}
\hline Inst & $Q_{z}$ & Synchs & Succs & $\Delta C_{\text {stoch }}[\%]$ & $\Delta C_{d e t}[\%]$ & $\Delta$ Time $[\%]$ & $\Delta$ Dist [\%] \\
\hline \multirow[t]{9}{*}{ n100_r2 } & 1 & 4.8 & 18.4 & -3.33 & 1.08 & 1.84 & 5.48 \\
\hline & 2 & 3.0 & 17.6 & -1.30 & 0.66 & 2.04 & 2.78 \\
\hline & 3 & 2.0 & 16.1 & -0.46 & 0.87 & 0.63 & 5.16 \\
\hline & 4 & 2.0 & 15.7 & -0.81 & 0.38 & 0.05 & 2.93 \\
\hline & 5 & 2.0 & 15.6 & -2.71 & 0.63 & 0.99 & 3.39 \\
\hline & 6 & 1.0 & 15.0 & -0.63 & 0.56 & 2.10 & 2.43 \\
\hline & 7 & 1.0 & 14.6 & -2.12 & 0.44 & 0.66 & 2.36 \\
\hline & 8 & 1.0 & 14.8 & -0.54 & 0.86 & 3.61 & 3.24 \\
\hline & 9 & 1.0 & 15.0 & -0.88 & 1.52 & 6.20 & 5.44 \\
\hline \multirow[t]{9}{*}{ n100_rc1 } & 1 & 7.0 & 23.2 & -3.55 & 0.98 & 0.90 & 5.91 \\
\hline & 2 & 5.0 & 21.6 & -1.38 & 0.72 & 2.71 & 2.79 \\
\hline & 3 & 3.4 & 20.3 & -3.05 & 0.55 & 1.81 & 2.27 \\
\hline & 4 & 3.0 & 20.0 & -1.74 & 0.94 & 2.64 & 4.54 \\
\hline & 5 & 2.0 & 17.9 & -2.12 & 0.69 & 2.33 & 2.64 \\
\hline & 6 & 2.0 & 17.8 & -1.51 & 2.39 & 10.53 & 7.48 \\
\hline & 7 & 2.0 & 18.2 & -0.55 & 1.63 & 5.18 & 6.82 \\
\hline & 8 & 2.0 & 17.6 & -2.93 & 1.21 & 4.65 & 4.86 \\
\hline & 9 & 2.0 & 17.5 & -2.53 & 0.82 & 1.80 & 4.19 \\
\hline \multirow[t]{9}{*}{ n100_rc2 } & 1 & 7.4 & 21.9 & -4.29 & 0.64 & 1.71 & 3.87 \\
\hline & 2 & 4.4 & 18.1 & -2.95 & 0.59 & 1.82 & 3.49 \\
\hline & 3 & 3.0 & 16.6 & -2.22 & 0.58 & 2.39 & 2.33 \\
\hline & 4 & 3.0 & 16.2 & -0.23 & 0.73 & 0.77 & 4.78 \\
\hline & 5 & 2.8 & 16.6 & -1.41 & 0.92 & 1.40 & 5.17 \\
\hline & 6 & 2.0 & 15.2 & -1.56 & 1.32 & 3.75 & 6.34 \\
\hline & 7 & 2.0 & 14.9 & -1.87 & 0.68 & 2.11 & 3.06 \\
\hline & 8 & 2.0 & 15.0 & -0.77 & 1.93 & 7.84 & 7.47 \\
\hline & 9 & 2.0 & 15.2 & -1.04 & 0.91 & 2.67 & 3.85 \\
\hline
\end{tabular}

Table 5 Detailed results for instances ' $\mathrm{n} 125 *$ '

\begin{tabular}{llllllll}
\hline Inst & $Q_{z}$ & Synchs & Succs & $\Delta C_{\text {stoch }}[\%]$ & $\Delta C_{\text {det }}[\%]$ & $\Delta$ Time $[\%]$ & $\Delta$ Dist $[\%]$ \\
\hline n125_c1 & 1 & 17.6 & 31.3 & -8.93 & 0.04 & 4.50 & -1.99 \\
& 2 & 11.8 & 31.8 & -6.72 & 0.81 & 0.86 & 4.44 \\
& 3 & 8.0 & 25.2 & -2.85 & 0.92 & 1.35 & 4.94 \\
& 4 & 6.4 & 23.7 & -3.25 & 1.23 & 2.45 & 7.24 \\
& 5 & 5.4 & 22.8 & -0.64 & 0.21 & 0.56 & 2.63 \\
& 6 & 4.0 & 20.6 & -1.80 & 0.33 & 1.46 & 0.60 \\
& 7 & 4.2 & 20.2 & -0.15 & 0.24 & 1.11 & 2.01 \\
& 8 & 3.6 & 20.5 & -1.42 & 0.31 & 1.69 & 1.51 \\
& 3.0 & 19.1 & -0.05 & 0.04 & 3.40 & -1.33 \\
\hline
\end{tabular}


Table 5 (continued)

\begin{tabular}{|c|c|c|c|c|c|c|c|}
\hline Inst & $Q_{z}$ & Synchs & Succs & $\Delta C_{\text {stoch }}[\%]$ & $\Delta C_{d e t}[\%]$ & $\Delta$ Time $[\%]$ & $\Delta$ Dist [\%] \\
\hline \multirow[t]{9}{*}{ n125_c2 } & 1 & 12.4 & 31.7 & -3.18 & 0.91 & 2.52 & 5.19 \\
\hline & 2 & 7.8 & 24.6 & -4.21 & 1.19 & 1.59 & 6.66 \\
\hline & 3 & 5.4 & 23.3 & -1.07 & 0.60 & 1.91 & 3.21 \\
\hline & 4 & 4.2 & 22.5 & -0.17 & 0.50 & 1.61 & 2.17 \\
\hline & 5 & 3.8 & 21.3 & -0.89 & 0.71 & 0.66 & 4.08 \\
\hline & 6 & 3.0 & 20.7 & -0.41 & 0.97 & 0.99 & 5.41 \\
\hline & 7 & 3.0 & 20.5 & -1.97 & 0.51 & 1.99 & 2.68 \\
\hline & 8 & 2.2 & 18.9 & -0.46 & 0.87 & 3.04 & 3.59 \\
\hline & 9 & 2.0 & 18.6 & -0.70 & 1.37 & 5.65 & 4.85 \\
\hline \multirow[t]{9}{*}{ n125_r1 } & 1 & 4.2 & 21.3 & -2.42 & 0.88 & 3.04 & 3.97 \\
\hline & 2 & 3.0 & 19.8 & -1.65 & 1.10 & 2.62 & 5.98 \\
\hline & 3 & 2.0 & 19.2 & -0.09 & 1.53 & 6.40 & 5.98 \\
\hline & 4 & 2.0 & 18.8 & -2.28 & 1.53 & 5.95 & 6.42 \\
\hline & 5 & 1.0 & 18.0 & -0.67 & 1.58 & 6.84 & 6.06 \\
\hline & 6 & 1.0 & 17.6 & -1.48 & 1.56 & 6.76 & 5.59 \\
\hline & 7 & 1.0 & 17.8 & -0.31 & 1.20 & 5.16 & 4.67 \\
\hline & 8 & 1.0 & 18.3 & -1.24 & 1.32 & 6.24 & 4.78 \\
\hline & 9 & 1.0 & 18.1 & -0.41 & 2.38 & 12.35 & 7.94 \\
\hline \multirow[t]{9}{*}{ n125_r2 } & 1 & 9.8 & 27.9 & -2.55 & 0.91 & 1.88 & 5.74 \\
\hline & 2 & 5.8 & 23.8 & -2.15 & 0.94 & 2.31 & 5.61 \\
\hline & 3 & 4.4 & 22.4 & -3.31 & 1.65 & 5.72 & 7.86 \\
\hline & 4 & 3.0 & 21.0 & -3.52 & 1.76 & 6.61 & 7.22 \\
\hline & 5 & 3.0 & 21.0 & -1.89 & 0.82 & 1.26 & 3.83 \\
\hline & 6 & 3.0 & 20.7 & -0.33 & 1.37 & 5.50 & 5.68 \\
\hline & 7 & 2.8 & 19.8 & -1.42 & 0.60 & 1.47 & 2.65 \\
\hline & 8 & 2.0 & 18.7 & -0.77 & 0.80 & 1.97 & 4.04 \\
\hline & 9 & 2.0 & 18.8 & -0.14 & 1.90 & 9.85 & 6.23 \\
\hline \multirow[t]{9}{*}{ n125_rc1 } & 1 & 6.2 & 24.1 & -2.44 & 0.82 & 0.63 & 4.85 \\
\hline & 2 & 4.0 & 21.3 & -2.56 & 1.07 & 2.99 & 5.34 \\
\hline & 3 & 3.0 & 20.9 & -2.66 & 0.46 & 0.95 & 2.70 \\
\hline & 4 & 2.0 & 19.5 & -0.91 & 1.27 & 3.93 & 5.42 \\
\hline & 5 & 2.0 & 19.2 & -1.97 & 1.36 & 4.33 & 5.89 \\
\hline & 6 & 2.0 & 19.0 & -1.59 & 1.34 & 4.71 & 5.44 \\
\hline & 7 & 2.0 & 18.7 & -1.90 & 1.43 & 4.56 & 6.24 \\
\hline & 8 & 1.0 & 17.5 & -0.58 & 1.39 & 5.85 & 4.96 \\
\hline & 9 & 1.0 & 17.7 & -1.81 & 0.35 & 1.59 & 1.68 \\
\hline \multirow[t]{9}{*}{ n125_rc2 } & 1 & 6.4 & 23.7 & -2.80 & 0.73 & 0.76 & 4.01 \\
\hline & 2 & 4.0 & 23.5 & -3.43 & 1.00 & 2.93 & 4.60 \\
\hline & 3 & 3.0 & 20.5 & -2.57 & 1.12 & 3.69 & 4.60 \\
\hline & 4 & 2.0 & 19.6 & -0.66 & 0.61 & 0.22 & 3.83 \\
\hline & 5 & 2.0 & 19.3 & -2.59 & 0.80 & 1.94 & 3.43 \\
\hline & 6 & 2.0 & 19.4 & -0.11 & 0.35 & 0.50 & 1.84 \\
\hline & 7 & 2.0 & 18.6 & -2.25 & 1.50 & 5.04 & 5.94 \\
\hline & 8 & 1.0 & 18.4 & -0.69 & 0.36 & 1.79 & 1.12 \\
\hline & 9 & 1.0 & 18.5 & -1.89 & 2.38 & 11.71 & 7.24 \\
\hline
\end{tabular}




\section{References}

Anderluh A, Hemmelmayr VC, Nolz PC (2017) Synchronizing vans and cargo bikes in a city distribution network. Cent Eur J Oper Res 25:345-376. https://doi.org/10.1007/s10100-016-0441-Z

Aron M, Bhouri N, Guessous Y (2014) Estimating travel time distribution for reliability analysis. In: Transportation research arena 2014

Bastian C, Rinnooy Kan AH (1992) The stochastic vehicle routing problem revisited. Eur J Oper Res 56(3):407-412. https://doi.org/10.1016/0377-2217(92)90323-2

Bertsimas DJ (1992) A vehicle routing problem with stochastic demand. Oper Res 40(3):574-585. https ://doi.org/10.1287/opre.40.3.574

Bertsimas DJ, van Ryzin G (1991) A stochastic and dynamic vehicle routing problem in the euclidean plane. Oper Res 39(4):601-615. https://doi.org/10.1287/opre.39.4.601

Boyles SD, Voruganti A, Waller ST (2010) Quantifying Travel Time Variability in Transportation Networks. Technical report, Center for Transportation Research University of Texas at Austin

Braekers K, Ramaekers K, van Nieuwenhuyse I (2016) The vehicle routing problem: state of the art classification and review. Comput Ind Eng 99:300-313. https://doi.org/10.1016/j.cie.2015.12.007

Cattaruzza D, Absi N, Feillet D, Gonzalez-Feliu J (2017) Vehicle routing problems for city logistics. EURO J Transp Logist 6:51-79

Chen L, Hà MH, Langevin A, Gendreau M (2014) Optimizing road network daily maintenance operations with stochastic service and travel times. Transp Res Part E Logist Transp Rev 64:88-102

Chen X, Feng L, Soon Ong Y (2012) A self-adaptive memeplexes robust search scheme for solving stochastic demands vehicle routing problem. Int J Syst Sci 43(7):1347-1366. https://doi. org/10.1080/00207721.2011.618646

Coelho BN, Coelho VN, Coelho IM, Ochi LS, K RH, Zuidema D, Lima MS, da Costa AR (2017) A multi-objective green uav routing problem. Comput Oper Res 88:306-315. https://doi.org/10.1016/j. cor.2017.04.011

Cuda R, Guastaroba G, Speranza M (2015) A survey on two-echelon routing problems. Comput Oper Res 55:185-199

Ehmke JF, Campbell AM, Urban TL (2015) Ensuring service levels in routing problems with time windows and stochastic travel times. Eur J Oper Res 240(2):539-550. https://doi.org/10.1016/j. ejor.2014.06.045

Erera AL, Morales JC, Savelsbergh M (2010) The vehicle routing problem with stochastic demand and duration constraints. Transp Sci 44(4):474-492. https://doi.org/10.1287/trsc.1100.0324

Gauvin C, Desaulniers G, Gendreau M (2014) A branch-cut-and-price algorithm for the vehicle routing problem with stochastic demands. Comput Oper Res 50:141-153. https://doi.org/10.1016/j. cor.2014.03.028

Gendreau M, Laporte G, Séguin R (1996) Stochastic vehicle routing. Eur J Oper Res 88(1):3-12. https:// doi.org/10.1016/0377-2217(95)00050-X

Gendreau M, Ghiani G, Guerriero E (2017) Time-dependent routing. Comput Oper Res 88:279. https:// doi.org/10.1016/j.cor.2017.08.003

Gomez A, Marino R, Akhavan-Tabatabaei R, Medaglia AL, Mendoza JE (2016) On modeling stochastic travel and service times in vehicle routing. Transp Sci 50(2):627-641. https://doi.org/10.1287/ trsc.2015.0601

Grangier P, Gendreau M, Lehuédé F, Rousseau LM (2016) An adaptive large neighborhood search for the two-echelon multiple-trip vehicle routing problem with satellite synchronization. Eur J Oper Res 254(1):80-91. https://doi.org/10.1016/j.ejor.2016.03.040

Huang Y, Zhao L, van Woensel T, Gross JP (2017) Time-dependent vehicle routing problem with path flexibility. Transp Res Part B: Methodol 95:169-195. https://doi.org/10.1016/j.trb.2016.10.013

Jensen P, Rouquier JB, Ovtracht N, Robardet C (2010) Characterizing the speed and paths of shared bicycle use in lyon. Transp Res Part D Transp Environ 15(8):522-524. https://doi.org/10.1016/j. trd.2010.07.002

Juan A, Faulin J, Grasman S, Riera D, Marull J, Mendez C (2011) Using safety stocks and simulation to solve the vehicle routing problem with stochastic demands. Transp Res Part C Emerg Technol 19(5):751-765. https://doi.org/10.1016/j.trc.2010.09.007

Lecluyse C, van Woensel T, Peremans H (2009) Vehicle routing with stochastic time-dependent travel times. 4OR 7(4):363-377. https://doi.org/10.1007/s 10288-009-0097-9 
Lei H, Laporte G, Guo B (2011) The capacitated vehicle routing problem with stochastic demands and time windows. Comput Oper Res 38(12):1775-1783. https://doi.org/10.1016/j.cor.2011.02.007

Leodolter M, Koller H, Straub M (2015) Estimating travel times from static map attributes. In: International Conference on Models and Technologies for Intelligent Transportation Systems (MT-ITS), IEEE, pp 121-126

Li X, Tian P, Leung SCH (2010) Vehicle routing problems with time windows and stochastic travel and service times: models and algorithm. Int J Prod Econ 125(1):137-145

Luo Z, Qin H, Zhang D, Lim A (2016) Adaptive large neighborhood search heuristics for the vehicle routing problem with stochastic demands and weight-related cost. Transp Res Part E: Logist Transp Rev 85:69-89. https://doi.org/10.1016/j.tre.2015.11.004

Mancini S (2017) A combined multistart random constructive heuristic and set partitioning based formulation for the vehicle routing problem with time dependent travel times. Comput Oper Res 88:290296. https://doi.org/10.1016/j.cor.2017.06.021

Minis I, Tatarakis A (2011) Stochastic single vehicle routing problem with delivery and pick up and a predefined customer sequence. Eur J Oper Res 213(1):37-51. https://doi.org/10.1016/j. ejor.2011.03.011

Montero A, Méndez-Díaz I, Miranda-Bront JJ (2017) An integer programming approach for the timedependent traveling salesman problem with time windows. Comput Oper Res 88:280-289. https:// doi.org/10.1016/j.cor.2017.06.026

Osman MS, Ram B (2017) Routing and scheduling on evacuation path networks using centralized hybrid approach. Comput Oper Res 88:332-339. https://doi.org/10.1016/j.cor.2017.06.022

Pillac V, Gendreau M, Guéret C, Medaglia AL (2013) A review of dynamic vehicle routing problems. Eur J Oper Res 225(1):1-11. https://doi.org/10.1016/j.ejor.2012.08.015

Psaraftis HN, Wen M, Kontovas CA (2016) Dynamic vehicle routing problems: three decades and counting. Networks 67(1):3-31. https://doi.org/10.1002/net.21628

Quak H, Balm S, Posthumus B (2014) Evaluation of city logistics solutions with business model analysis. Proc-Soc Behav Sci 125:111-124

Rahmani M, Jenelius E, Koutsopoulos HN (2015) Non-parametric estimation of route travel time distributions from low-frequency floating car data. Transp Res Part C Emerg Technol 58(Part B):343362. https://doi.org/10.1016/j.trc.2015.01.015, big Data in Transportation and Traffic Engineering

Soysal M, Cimen M (2017) A simulation based restricted dynamic programming approach for the green time dependent vehicle routing problem. Comput Oper Res 88:297-305. https://doi.org/10.1016/j. cor.2017.06.023

Soysal M, Bloemhof-Ruwaard JM, Bektas T (2015) The time-dependent two-echelon capacitated vehicle routing problem with environmental considerations. Int J Prod Econ 164:366-378. https://doi. org/10.1016/j.ijpe.2014.11.016

Stewart WR, Golden BL (1983) Stochastic vehicle routing: a comprehensive approach. Eur J Oper Res 14(4):371-385. https://doi.org/10.1016/0377-2217(83)90237-0

Taş D, Gendreau M, Dellaert N, van Woensel T, de Kok AG (2014) Vehicle routing with soft time windows and stochastic travel times: a column generation and branch-and-price solution approach. Eur J Oper Res 236(3):789-799. https://doi.org/10.1016/j.ejor.2013.05.024

Tavakkoli-Moghaddam R, Raziei Z (2016) A new bi-objective location-routing-inventory problem with fuzzy demands. In: 8th IFAC conference on manufacturing modelling, management and control MIM, IFAC-PapersOnLine 49(12):1116 - 1121. https://doi.org/10.1016/j.ifacol.2016.07.646

Toplak W, Asamer J, Straub M, Dragaschnig M, Puchinger J, Prandtstetter M (2011) Route analysis and planning for urban transportation on basis of floating car data and emission estimates. In: Transportation research board 90th annual meeting, 11-1804

Wang X, Crainic TG, Wallace SW (2016) Stochastic scheduled service network design: the value of deterministic solutions. Technical report, CIRRELT

WHO (2016) Air pollution levels rising in many of the world's poorest cities

Zhang J, Lam WHK, Chen BY (2013) A stochastic vehicle routing problem with travel time uncertainty: trade-off between cost and customer service. Netw Spatial Econ 13(4):471-496. https://doi. org/10.1007/s11067-013-9190-x

Zhang T, Chaovalitwongse WA, Zhang Y (2012) Scatter search for the stochastic travel-time vehicle routing problem with simultaneous pick-ups and deliveries. Comput Oper Res 39(10):2277-2290. https ://doi.org/10.1016/j.cor.2011.11.021 
Publisher's Note Springer Nature remains neutral with regard to jurisdictional claims in published maps and institutional affiliations.

Alexandra Anderluh is researcher at the Research Institute for Supply Chain Management at WU Vienna. Her research interests include advanced routing problems in the context of sustainable urban logistics. She continuously supplements her scientific knowledge with national and international projects, including the CONCOORD project. Her recent publications focus on the integration of emission-free vehicles like cargo bikes into urban distribution schemes.

Rune Larsen is Associate Professor at DTU Management at the Technical University of Denmark. He holds a Ph.D. from the University of Southern Denmark. His research interests are focussed on dynamic and stochastic problems, typically with real life applications.

Vera C. Hemmelmayr is Associate Professor at the Institute of Transport and Logistics Management at the Vienna University of Economics and Business (WU). She holds a Ph.D. from the University of Vienna and a habilitation from WU. Before joining WU, she worked at the University of Vienna and at the CIRRELT (Montreal) as a postdoctoral fellow. Her research interests include models and solution methods for optimization problems in green logistics.

Pamela C. Nolz is scientist at the Center for Mobility Systems at AIT Austrian Institute of Technology. During her Ph.D. studies she worked at the University of Vienna and the Ecole Centrale de Lille. After receiving her Ph.D. from the University of Vienna, she joined the Ecole des Mines de Saint-Etienne as a postdoctoral researcher. Before working at AIT, she was assistant professor at the Institute for Production Management at WU Vienna University of Economics and Business. Her research interests are focused on operations research in sustainable and humanitarian logistics. 